\title{
A new patch technique for valve-sparing aortic root repair in acute type A aortic dissection
}

\author{
Ling-Chen Huang, Zheng Xu, Xiao-Fu Dai \\ Department of Cardiovascular Surgery, Union Hospital, Fujian Medical University, Fuzhou, China \\ Correspondence to: Xiao-Fu Dai. Department of Cardiovascular Surgery, Union Hospital, Fujian Medical University, Fuzhou 350001, China. \\ Email: 1470054258@qq.com.
}

\begin{abstract}
Valve-sparing aortic root replacement is an attractive option for younger patients with acute type A aortic dissection. This study aimed to design a new patch technique for reconstructing the aortic root and preserving the aortic valve following aortic dissection. Between July 2017 and December 2018, 35 patients underwent valve-sparing aortic root repair using this new patch technique. All participants were in the supine position, transesophageal echocardiography and median sternotomy were routinely performed. After thrombi at the aortic root were removed in acute type A dissection, the luminal aortic intimal dissection was removed until the aortic condition was normalized. In each aortic sinus involved in the dissection, a Dacron-graft patch with the shape corresponding to the defect was sutured to the normal remnant vascular wall or aortic annulus in the aortic sinus using 5-0 Prolene suture to reconstruct the aortic root. A total of 2 patients died, and 1 cerebral infarction, and 3 cases of transient brain dysfunction were recorded. The sinus tube junction and sinus diameter were within the normal ranges when they were reexamined 3 months after surgery. This new patch technique circumvents the redesign of the spatial $3 \mathrm{D}$ structure of the aortic valve, is simple to operate, and easy to master. It completely removes the diseased dissection tissue, avoids the use of glue, and is an alternative surgical technique, especially for beginners.
\end{abstract}

Keywords: Aortic dissection; valve-sparing aortic root repair; new patch technique

Submitted Jan 07, 2021. Accepted for publication Apr 29, 2021.

doi: $10.21037 / \mathrm{atm}-21-436$

View this article at: http://dx.doi.org/10.21037/atm-21-436

\section{Introduction}

Acute type A aortic dissection is a life-threatening medical condition associated with extremely high rates of mortality and morbidity, and its diagnosis and treatment remain a challenge $(1,2)$. Patients with acute Stanford type A aortic dissection who do not receive treatment die at a rate of $1-2 \%$ per hour during the first day and almost half die by 1 week. The surgical treatment for type A aortic dissection aims to remove the entry into the false lumen and reconstitute the aortic true lumen with a synthetic interposition graft with or without reimplantation of the coronary arteries. The surgical treatment of aortic root is also of great importance. In addition, restoration of aortic valve competence by valvesparing techniques appeared to be more physiologic when compared with the reimplantation technique (3).
Valve-sparing aortic root replacement (VSRR) is an attractive option for younger patients with acute type A aortic dissection (4). The David procedure or Urbanski patch technique, as a VSRR, has been applied in many centres and achieved good mid- and long-term outcomes, but it is time-consuming and complicated (5). Once bleeding occurs, it is difficult to control, and newly qualified surgeons have difficulty mastering these procedures. To overcome these shortcomings, we designed a new patch technique using a Dacron vascular patch to repair the dissection, reconstruct the aortic root, and preserve the aortic valve. In this study, according to the aortic root morphology, the intimal dissection sheets at the root are removed except the normal vessel wall or annulus tissue, and the missing portion is repaired with a Dacron-graft patch of the corresponding shape to reconstruct the aortic 
A

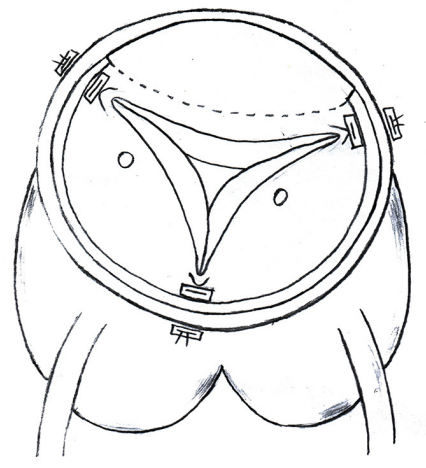

C

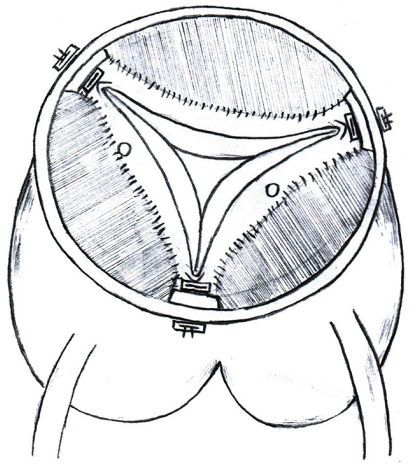

B

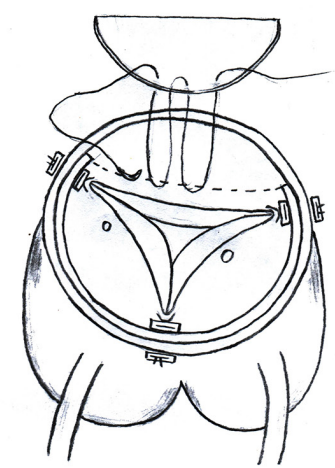

D

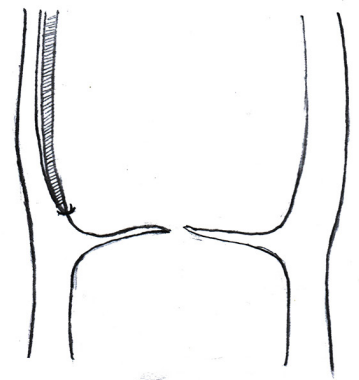

Figure 1 (A,B,C,D) Schematic diagram of the new patch technique procedure. The commissures of the leaflets were sutured to resuspend the aortic valve and the dissection sheets at the root was incised except the normal vessel wall or annulus tissue (A). The incised portion is repaired with a Dacron-graft patch of the corresponding shape to reconstruct the aortic root (B). Treatment of the left and right coronary sinus involvement according to the Neri classification (C and D).

root. If the coronary arteries in the left and right coronary sinus were involved, they were treated according to the Neri classification. Different classification has varying treatment strategies. We present the following article in accordance with the MDAR checklist (available at http:// dx.doi.org/10.21037/atm-21-436) (6).

\section{Methods}

Between July 2017 and December 2018, 35 patients underwent valve-sparing aortic root repair using this new patch technique. All participants were in the supine position, transesophageal echocardiography and median sternotomy were routinely performed, and cardiopulmonary bypass was established through the femoral artery, axillary artery, and superior and inferior vena cava. The ascending aorta was cross-clamped, and the left and right coronary arteries were directly perfused with cold blood cardioplegic solution. The ascending aorta was longitudinally dissected up to $0.5 \mathrm{~cm}$ above the border of the right noncoronary sinus, and the adventitia of the ascending aorta was lifted. All procedures were performed in the lumen. Thrombi at the aortic root were removed, and three pledged 4-0 polypropylene mattress sutures at the top of the three commissures were used to resuspend the aortic valve (Figure 1A). The luminal aortic intimal dissection was removed until the aortic was healthy. In each aortic sinus involved in the dissection, a Dacron-graft patch with the shape corresponding to the defect was sutured to the normal remnant vascular wall or aortic annulus in the aortic sinus using 5-0 Prolene suture to reconstruct the aortic root (Figure 1B). If the coronary arteries in the left and right coronary sinus were involved, they were treated according to 
Table 1 Aortic incompetence (No. of patients)

\begin{tabular}{lccccc}
\hline \multirow{2}{*}{ Time } & \multicolumn{5}{c}{ Aortic insufficiency } \\
\cline { 2 - 6 } & 0 & 1 & 2 & 3 & 4 \\
\hline Before surgery & 2 & 3 & 15 & 12 & 3 \\
Before discharge & 26 & 4 & 2 & 1 & 0 \\
3 months after surgery & 24 & 5 & 3 & 1 & 0 \\
\hline
\end{tabular}

the Neri classification (Figure 1C,D). For type A, the intimal dissection tissue was removed to the normal coronary ostium, and the corresponding Dacron-graft patch was sutured to the normal coronary ostium and sinus wall. For type B, the intimal dissection tissue around the coronary ostium was removed, a felt piece was placed between the inner and outer dissection sheets at the coronary artery ostium, and a Dacron-graft patch with the corresponding shape was sutured to the coronary ostium. For type $\mathrm{C}$, the coronary ostium was closed, and coronary artery bypass surgery was performed. Therefore, the new sinus tube junction was composed of the Dacron-graft patch and normal sinus wall, then a Dacrongraft tube was used to replace the ascending aorta. Once the temperature of the nasopharyngeal had decreased to $20^{\circ} \mathrm{C}$, the ascending aortic clamp was removed, vascular clamps were applied to the innominate and left common carotid arteries, antegrade cerebral perfusion was initiated via the axillary artery at $10-15 \mathrm{~mL} / \mathrm{kg} / \mathrm{min}$, and reconstruction or replacement of the distal vessel were performed. Drainage of the aortic root and right atrium was performed routinely. The study was conducted in accordance with the Declaration of Helsinki (as revised in 2013). This study was approved and monitored by the ethics committee of the Fujian Union Hospital and individual consent for this retrospective analysis was waived.

\section{Results}

From June 2017 to December 2018, a total of 35 patients (23 males, mean age $45.6 \pm 21.6$ years, range $33-72$ years) with acute type $\mathrm{A}$ aortic dissection underwent valve-sparing aortic dissection repair using a Dacron-graft patch. The time from onset to surgery was 3-76 hours (mean: $18.2 \pm 10.2$ hours). The cardiopulmonary bypass time was $120-320$ min (mean: $140 \pm 38 \mathrm{~min})$. The aortic cross-clamp time was $45-124 \mathrm{~min}$ (mean: $92 \pm 27 \mathrm{~min}$ ), and the selective cerebral perfusion time was $19-42$ min (mean: $23.2 \pm 4.9 \mathrm{~min}$ ). Pericardial drainage volume was $95-650 \mathrm{~mL}$ (mean: $130 \pm 75 \mathrm{~mL}$ ). Among the
35 participants, there were 2 deaths, 1 cerebral infarction, and 3 cases of transient brain dysfunction. The sinus tube junction and sinus diameter were within the normal ranges $(28.1 \pm 4.5$ and $30.2 \pm 3.4 \mathrm{~mm}$, respectively) at the postoperative 3 -month follow-up on aortic computed tomography angiography (CTA) scans. The monitoring data of preoperative and postoperative aortic regurgitation are listed in Table 1.

\section{Discussion}

The treatment of the aortic root in acute type A aortic dissection is mainly focused on aortic regurgitation and reconstruction of the sinus involved in the dissection. The majority of aortic regurgitation is caused by detachment of the aortic valve commissure, while the structure of the valve itself has no lesions $(3,7)$. Root surgery strategies that preserve the aortic valve have been gradually recognized by many scholars. Currently, there are many modified David procedures, such as Urbanski's patch technique (8) and Rylski's neomedia technique (9).

Our approach is to remove the thrombus and resuspend the aortic valve commissure to eliminate aortic regurgitation. According to the original aortic root morphology, all the intimal dissection sheets at the root are removed except the normal vessel wall or annulus tissue, and the missing portion is repaired with a Dacron-graft patch of the corresponding shape to reconstruct the aortic root. For Neri type A cases involving the coronary artery, the intimal dissection sheet close to the coronary artery can be completely removed, and a Dacron-graft patch with the corresponding shape is used for repair. For type $\mathrm{B}$ cases involving the coronary artery, the intimal sheet at the coronary ostium can be kept in a button shape, a felt sheet is lined between the inner and outer membrane layer, and it is repaired with an artificial vascular patch of the corresponding shape. Therefore, the entire aortic root is composed of normal vascular wall without dissection or a Dacron-graft patch, which completely eliminates the diseased dissection tissue.

Compared with other VSRR procedures, our technique is simple. The repairs and sutures are based on the shape of the aortic root itself without redesign of the spatial three-dimensional (3D) structure of the aortic valve. Compared with Urbanski's patch technique, only the diseased sinus dissection tissue is removed, and coronary artery transplantation is not required. In contrast with Rylski's neomedia technique, the diseased dissection tissue 
is completely removed, and the use of glue is avoided. All the sutures are performed in the lumen to anastomose with normal tissues or the Dacron-graft patch. Each suture involves a small portion of the outer membrane, which reduces the probability of bleeding. If there is still nonsurgical oozing after heparin neutralization with protamine, a drainage fistula between the ascending aortic root and right atrium would be performed, which greatly shortens the operation time.

In summary, reconstruction of the aortic root using a Dacron patch overcomes the disadvantages of life-long anticoagulation. VSRR technique solves the problems of pseudoaneurysm, root dilatation and aortic regurgitation in the proximal anastomosis. In additional, this technique circumvents the redesign of the spatial 3D structure of the aortic valve, is simple to operate, and easy to master. It completely removes the diseased dissection tissue, avoids the use of glue, and is an alternative surgical technique, especially for beginners.

\section{Acknowledgments}

Funding: This study was supported by the Funds for the Innovation of Medicine, Fujian Province (2016-CX-25).

\section{Footnote}

Reporting Checklist: The authors have completed the MDAR checklist. Available at http://dx.doi.org/10.21037/atm-21436

Data Sharing Statement: Available at http://dx.doi. org/10.21037/atm-21-436

Conflicts of Interest: All authors have completed the ICMJE uniform disclosure form (available at http:// dx.doi.org/10.21037/atm-21-436). The authors report grants from The Funds for the Innovation of Medicine, Fujian Province [grant number 2016-CX-25], outside the submitted work. The authors have no other conflicts of interest to declare.

Ethical Statement: The authors are accountable for all aspects of the work in ensuring that questions related to the accuracy or integrity of any part of the work are appropriately investigated and resolved. The study was conducted in accordance with the Declaration of Helsinki (as revised in 2013). This study was approved and monitored by the ethics committee of the Fujian Union Hospital and individual consent for this retrospective analysis was waived.

Open Access Statement: This is an Open Access article distributed in accordance with the Creative Commons Attribution-NonCommercial-NoDerivs 4.0 International License (CC BY-NC-ND 4.0), which permits the noncommercial replication and distribution of the article with the strict proviso that no changes or edits are made and the original work is properly cited (including links to both the formal publication through the relevant DOI and the license). See: https://creativecommons.org/licenses/by-nc$\mathrm{nd} / 4.0 \%$.

\section{References}

1. Evangelista A, Isselbacher EM, Bossone E, et al. Insights From the International Registry of Acute Aortic Dissection: A 20-Year Experience of Collaborative Clinical Research. Circulation 2018;137:1846-60.

2. Pape LA, Awais M, Woznicki EM, et al. Presentation, Diagnosis, and Outcomes of Acute Aortic Dissection: 17-Year Trends From the International Registry of Acute Aortic Dissection. J Am Coll Cardiol 2015;66:350-8.

3. Nienaber CA, Clough RE. Management of acute aortic dissection. Lancet 2015;385:800-11.

4. David TE. The aortic valve-sparing operation. J Thorac Cardiovasc Surg 2011;141:613-5.

5. Leshnower BG, Myung RJ, McPherson L, et al. Midterm results of David $\mathrm{V}$ valve-sparing aortic root replacement in acute type A aortic dicssection. Ann Thorac Surg 2015;99:795-800; discussion 800-1.

6. Macleod M, Collings AM, Graf C, et al. The MDAR (Materials Design Analysis Reporting) Framework for transparent reporting in the life sciences. Proc Natl Acad Sci U S A 2021;118:e2103238118.

7. Hiratzka LF, Bakris GL, Beckman JA, et al. 2010 ACCF/ AHA/AATS/ACR/ASA/SCA/SCAI/SIR/STS/SVM Guidelines for the diagnosis and management of patients with thoracic aortic disease. A Report of the ACCF/AHA/ AATS/ACR/ASA/SCA/SCAI/SIR/STS/SVM. J Am Coll Cardiol 2010;55:e27-e129. 
8. Urbanski PP. Valve-sparing aortic root repair with patch technique. Ann Thorac Surg 2005;80:839-43.

9. Rylski B, Bavaria JE, Milewski RK, et al. Long-term results of neomedia sinus valsalva repair in 489 patients with

Cite this article as: Huang LC, Xu Z, Dai XF. A new patch technique for valve-sparing aortic root repair in acute type A aortic dissection. Ann Transl Med 2021;9(11):949. doi: 10.21037/atm-21-436 type A aortic dissection. Ann Thorac Surg 2014;98:582-8; discussion 588-9.

(English Language Editor: J. Jones) 